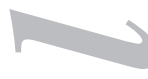

\title{
Dispepsia funcional y Helicobacter pylori: ¿convivencia estable?
}

La publicación del trabajo en este número de la revista, cuyo título resumido: "Dispepsia funcional y Helicobacter pylori: respuesta al tratamiento", de los autores Artaza Varasa y cols., me permite este artículo editorial en el que expondré algunas reflexiones personales sobre este síndrome digestivo, tan antiguo, que ya fue mencionado por Hipócrates.

Mi reflexión primera abarca los problemas conceptuales y los mecanismos etiopatogénicos y fisiopatológicos. Respecto al concepto, con el paso del tiempo han ocurrido modificaciones conceptuales y definitorias paralelas al cambio del pensamiento médico sobre las enfermedad en general y sobre las enfermedades digestivas, en particular, consecuentes con los avances logrados merced a la investigación de las posibles causas, así como a la aplicación de sensibles y certeros métodos diagnósticos y fármacos de probada eficacia. Sin embargo, siguen vigentes algunos de los pensamientos de los médicos clínicos de comienzos del siglo XX.

Algunos apuntes: en la $2^{a}$ edición del Tratado de Patología Médica, de Sargent, publicado en 1927, inicia la sección de "Dispepsias gástricas" con estas palabras: "El capítulo de las dispepsias ha contenido toda la patología gástrica hasta el día en que la práctica de las necropsias ha aportado sólidas bases para la interpretación de los síntomas morbosos" (1). Dedica a esta dolencia 50 páginas. Menciona subgrupos de dispepsias idiopáticas y nerviosas.

Luis Urrutia publica en 1925 la 2 a edición de su Tratado de Enfermedades del estómago, primer libro español, monográfico sobre el tema. Su capítulo sobre "Afecciones nerviosas del estómago" incluye los subgrupos de "Dispepsia nerviosa y dispepsia psicógena" con un concepto, en cierta forma, profético. "La dispepsia nerviosa no es una afección gástrica ni una neurosis especial localizada en el estómago. Los enfermos que la padecen son neuróticos que localizan preferentemente sus molestias en el estómago (o en los intestinos) en vez de hacerlo en otro órgano cualquiera" (2).

Ha transcurrido casi un siglo en el que han ocurrido importantes avances etiológicos, diagnósticos y terapéuticos (la bacteria Helicobacter pylori, las diversas modalidades de diagnóstico de imagen, especialmente la endoscopia digestiva y metodología de la funcionalidad digestiva, los antisecretores bloqueantes $\mathrm{H} 2$ e inhibidores de la bomba de protones -IBP-) que han modificado profundamente la conducción clínica de los enfermos digestivos.

¿Ha cambiado el concepto de dispepsia? En 1997, Mearin F, dirige una monografía con el título: Dispepsia funcional: tan desconocida como frecuente. Intenta una definición racional sobre bases rigurosamente científicas. Afirma que debe estar relacionada con "trastornos de la función del tracto digestivo superior o debida a una percepción anómala del paciente" (3). Excluye a los pacientes con infección de 
Helicobacter pylori y a los pacientes "pesados" (entiendo que se refiere a los neuróticos). Subdivide los pacientes en tres categorías: tipo úlcera, tipo dismotilidad y tipo inespecífico.

En 2007, la Sociedad Española de Patología Digestiva ha sumado a la colección de su Biblioteca básica una reciente Monografía sobre Dispepsia funcional. El mismo autor, después de 10 años, titula el capítulo conceptual con interrogación "¿Qué se entiende por dispepsia orgánica y funcional?" (4). Incluye la definición Roma III: "síntomas o conjunto de síntomas que la mayoría de médicos consideran tienen su origen en la región gastroduodenal". Mantiene la misma subdivisión, aunque afirma que no es válida en la práctica clínica. Incluye el síndrome de distrés postprandial y de dolor epigástrico. Habla de la hipersensibilidad visceral gástrica con aumento de la percepción. Todo ello señala la indefinición conceptual lo que justifica el acierto del autor al incluir el título entre interrogantes.

La respuesta a la pregunta es que no ha cambiado el concepto. Las diferentes definiciones son como variaciones a un mismo tema inconcreto, de límites imprecisos en el que se intenta convertir lo "subjetivo" en "objetivo"; localizar y responsabilizar las molestias percibidas por el paciente a anormalidades "funcionales" del estómago-duodeno o del "segmento digestivo superior". El intento ha dado sus frutos prácticos en cuanto que ha hecho posible prescripciones terapéuticas con alivio de los síntomas, aunque en muchos pacientes de forma temporal.

¿A qué se debe esta respuesta temporal del tratamiento? Aporto varias posibles respuestas. La más sencilla: el inadecuado diagnóstico impide realizar el adecuado tratamiento. Ahora bien, en las consultas diarias, pocos médicos de Atención Primaria (AP) tienen en su mesa de trabajo las conclusiones del comité de Roma II de 1999, muy rígido en cuanto a limitar la duración y otras características; ni el de Roma III, en el que con un sentido más práctico enuncia recomendaciones diferenciadas para la práctica clínica y para los ensayos clínicos $(5,6)$. La mayoría de estos médicos limitan su trabajo a tratar los síntomas del paciente con IBP o con procinéticos. Algunos con acceso libre a las pruebas de diagnóstico endoscópico recomiendan panendoscopia oral. Otros solicitan pruebas diagnósticas de Helicobacter, con preferencia la prueba de aliento con urea marcada con C13. No disponen de otras complejas técnicas diagnósticas de disfunciones motoras o de percepción de la sensibilidad.

Puede fallar el tratamiento o resultar temporalmente eficaz, porque bastantes pacientes padecen solapamiento de los síntomas de la DF con los del síndrome de colon o intestino irritable (SII), con aerofagia, eructos y con otros trastornos funcionales. Además, los síntomas del síndrome de DF pueden experimentar un cambio sindrómico evolutivo a síntomas más específicos de SII. Numerosos estudios han aportado datos que confirman estas afirmaciones.

En 1995, el grupo sueco de Lars Agréus y cols. investiga en 1.290 individuos de la población general el padecimiento de trastornos funcionales con un cuestionario adecuado y el cambio experimentado durante un año. De los datos obtenidos destacan que el $87 \%$ de pacientes con SII cumplían los criterios diagnósticos de dispepsia. El solapamiento entre los distintos subgrupos de dispepsia alcanzaba el 50\%. Al cabo de 1 año de seguimiento el 50\% modificaban su perfil sintomático (7). Los autores concluyen que la separación diagnóstica de los síndromes funcionales puede ser inapropiada por el solapamiento, por la alternancia y porque pueden ser originados por una alteración de la percepción visceral. En esta línea, Lindell y cols. (8) comprobaron que a los 10 años de seguimiento, el 30\% de pacientes con DF han padecido síntomas compatibles con SII. 
Otros muchos estudios avalan estos datos. En España, en un estudio poblacional, los investigadores hallaron solapamiento de SII y de DF en el 50\% y de DF con SII en el 30\% de los individuos encuestados (9). Recientemente, Conchillo y cols. (10), aplicando pH-metría e impedancia, comprueban una mayor incidencia de reflujo gastroesofágico gaseoso (no-ácido) en pacientes con DF que en el grupo control.

¿Cómo interpretar estos datos? ¿Qué significan? Muy acertadamente, en un artículo crítico, Rey Díaz-Rubio explica y justifica esta asociación de los síndromes funcionales digestivos, porque: "comparten factores etiológicos y fisiopatológicos: alteraciones de la motilidad, de la sensibilidad del tubo digestivo, factores psicosociales e incluso algunos infecciosos" (11). Personalmente, coincido con esta opinión y estoy seguro que la comparten la mayoría de los médicos de AP que atienden pacientes con DF. Tenía razón Urrutia al afirmar hace casi cien años que "la dispepsia nerviosa no es una afección gástrica..."

La bacteria Helicobacter pylori $(\mathrm{Hp})$ es el otro consorte del matrimonio con DF. ¿Qué ha sucedido en los casi 25 años de convivencia? Destaco algunos de los hechos suficientemente probados y aceptados por la inmensa mayoría de los médicos y por la comunidad científica, al principio escéptica, pero rendida después a la evidencia de la abrumadora cifra de datos confirmadores de que la bacteria $\mathrm{Hp}$ no era "comensal inocuo", ni huésped "saprofito" de la mucosa gástrica, sino un agente infeccioso relacionado, directamente, con la mayoría de la enfermedades gástricas y, de forma indirecta, con algunas enfermedades extradigestivas. Ha supuesto un cambio radical en los conceptos, en el diagnóstico y sobre todo, en el tratamiento de la úlcera péptica, que, por primera vez en la historia puede hablarse de enfermedad curable. Los descubridores Warren y Marshall recibieron el premio Nobel por su descubrimiento en el año 2005 lo que supuso el reconocimiento mundial a sus aportaciones para mejorar las dolencias digestivas.

Es un hecho comprobado que la infección por $\mathrm{Hp}$ de la mucosa gástrica causa gastritis crónica de mayor o menor cuantía en los pacientes infectados con posibilidades evolutivas a lesiones premalignas de gastritis atrófica (GA), metaplasia intestinal (MI) y displasia. Pero, ¿qué papel juega en la DF? ¿Se debe diagnosticar en la DF? ¿Se deben tratar todos los pacientes de DF infectados por $H p$ ? ¿Disponemos de métodos diagnósticos idóneos para seleccionar el grupo de DF infectado susceptible de tratamiento erradicador? Algunas de estas preguntas han sido contestadas, con argumentos y datos científicos convincentes para los médicos clínicos y para los investigadores del tema, de modo satisfactorio. La contestación a otras se apoya en datos menos convincentes. Intentaré exponer mi personal punto de vista desde la perspectiva "confesional" de haber defendido, desde hace más de 20 años la eliminación de la bacteria $H p$ en pacientes con DF.

En la línea de reflexión inicial, la infección de $H p$ no es la causa de DF porque la misma definición excluye cualquier factor de organicidad. Es un factor más asociado a las manifestaciones clínicas del síndrome. Estudios pioneros demostraron que la prevalencia de $H p$ en pacientes con dispepsia no ulcerosa (DNU), denominación antigua de la DF, era similar a la de la población general asintomática (12). Hay una carencia de síntomas específicos y una indefinición del perfil sintomático de pacientes infectados (13). La respuesta al tratamiento con la desaparición de los síntomas resultaba controvertida: algunos estudios demostraron mejoría de los síntomas (14), otros comprobaron que los síntomas persistían tras la desaparición de la bacteria (15). 
Desde aquellas lejanas fechas hasta la actualidad, centenares de trabajos mantienen las mismas dudas.

La infección de $H p$ causa gastritis con lesiones histológicas de intensidad y potencialidad evolutiva variables, como he comentado. Se ha intentado relacionar la aparición de síntomas y la consiguiente respuesta al tratamiento erradicador con el grado de gastritis antral. De hecho es uno de los objetivos de los autores del artículo publicado en este número. No es nuevo este afán. Desde el principio de la era del $\mathrm{Hp}$, se intentó relacionar los síntomas y la tasa de respuesta al tratamiento erradicador con el grado de actividad de la gastritis obteniéndose resultados contradictorios $(16,17)$. Los autores del trabajo, objeto de este artículo editorial, aportan datos en los que parece que los pacientes con mayor grado de actividad de la gastritis antral obtienen mayor beneficio de la erradicación de $\mathrm{Hp}$. Es una lástima que no hayan obtenido biopsias duodenales para conocer la histología de la mucosa del duodeno de los pacientes con gastritis antral infectado por $\mathrm{Hp}$. Es posible que algunos tuvieran duodenitis y metaplasia gástrica, colonizada por $\mathrm{Hp}$, lo que hubiera sido una prueba de que, en realidad, estos pacientes eran potenciales ulcerosos duodenales. Otro problema adicional aún no resuelto que resta validez a la subdivisión en grupos afines de los pacientes con DF.

En 1997, exponía una hipótesis sobre la posible participación del Hp en la producción de síntomas sobre la base de pilares orgánicos: inflamación de la mucosa gastroduodenal con producción de citocinas, neuropéptidos y otros factores, hipersecreción ácida, alteración de la motilidad gastroduodenal, hipersensibilidad gastroduodenal con aumento de la percepción (18). ¿Se ha logrado evidencia científica que documente la organicidad fisiopatológica de la infección de $H p$ en la producción de síntomas de la DF?

Estudios aislados han proporcionado datos controvertidos, aunque, la mayoría negativos. Recientemente Calvet Calvo (19), en una revisión sistemática rigurosa por PubMed y otras fuentes bibliográficas, concluye que la mayoría de los estudios no han comprobado modificaciones en el vaciamiento y motilidad gástrica; que algunos estudios han demostrado aumento de la sensibilidad visceral en respuesta a la distensión gástrica medida por barostato; y que los cambios de la secreción gástrica no inducen síntomas en la DF. Sin embargo, Moayyedi y cols. (20) en un minucioso metaanálisis de numerosos estudios de la acción del tratamiento erradicador sobre los síntomas de la DF demuestran un efecto curativo duradero en uno de cada 18 pacientes tratados.

La controversia respecto al diagnóstico y tratamiento del $H p$ en pacientes con DF ha existido desde el descubrimiento de la bacteria. Los médicos que creíamos en el papel patógeno de la bacteria, diagnosticábamos y tratábamos a los pacientes con DF e infectados por $H p$, sin una sólida evidencia científica, pero con el aval del propio paciente que aceptaba la propuesta del tratamiento erradicador a pesar de la falta de evidencia científica sobre su efectividad. Como los medios de comunicación publicaban noticias sobre la bacteria $\mathrm{Hp}$, muchos pacientes que acudían a la consulta con molestias digestivas exigían la prueba del $\mathrm{Hp}$. Si resultaba positiva, nos reclamaban el tratamiento. Petición no rechazada por los médicos convencidos de que la bacteria era la causante de una gastritis con una evolución natural indeterminada. Con esta idea y pensamiento, ¿para qué dejar la bacteria? Su mejor destino debería ser su muerte y su desaparición con el tratamiento.

La continuada investigación sobre este tema ha producido datos que han permitido disipar algunas y fortalecer las débiles evidencias científicas, lo que ha contribuido a modificar la opinión y la conducta de los médicos para diagnosticar y tratar a 
los pacientes con DF. En efecto, el primer Consenso de Expertos organizado por el Instituto Nacional de la Salud de Estados Unidos, en 1994, contraindicaba el diagnóstico de $H p$ en DNU y consiguiente tratamiento de los infectados (21). Un posterior Consenso del Grupo Europeo para estudio de Helicobacter, en 1997, aconsejaba el diagnóstico y el tratamiento a pesar de la escasa evidencia y controvertidos datos existentes (22). El Consenso de Roma III sobre trastornos funcionales digestivos (23), al referirse a la DF, incorpora la diferencia entre DF investigada y no investigada. Exige para el diagnóstico de DF la exclusión de la infección $H p$. Para el diagnóstico de DF no investigada con panendoscopia en pacientes menores de 50 años sin síntomas "de alarma", aconseja una prueba no invasiva (prueba de aliento con urea, antígeno en heces) para $\mathrm{Hp}$ y realizar tratamiento erradicador en los infectados. Esta táctica de "diagnosticar y tratar" (test and treat) ha demostrado ser la de mayor utilidad en cuanto a coste-efectividad.

Hay otras razones para diagnosticar la presencia de $H p$ y tratar los infectados en pacientes con DF. Unas son económicas porque evita prolongados tratamientos sintomáticos y reduce las visitas a la consulta del médico de AP y del especialista. Para demostrarlo, Ford y cols. (24) realizaron un ensayo clínico consistente en un "cribado" de la población general con test de $H p$. Seleccionaron un grupo de 8.407 sujetos, menores de 50 años, que no padecían síntomas de DF. Los que resultaron positivos fueron tratados con terapia erradicadora. De los $H p$ negativos, un subgrupo recibió tratamiento placebo, otro no recibió tratamiento alguno ni información del estatus de $H p$ y un tercer subgrupo no recibió tratamiento, pero sabían que no estaban infectados. Al cabo de dos años, los pacientes $H p$ positivos curados y los $H p$ negativos conocedores de su estado bacteriano gastaron menos en recursos sanitarios. Aunque resultara un ahorro reducido, los autores sugieren que puede ser mayor a los 10 años, hecho demostrado con pacientes $H p$ positivos en otro ensayo clínico (25).

Por último, otra indicación de diagnosticar y tratar infección de $H p$ en pacientes con DF es la prevención de la patología gastroduodenal asociada. Las lesiones de la gastritis crónica por $H p$ contienen un potencial evolutivo en cuyo desarrollo participan factores de la propia bacteria, del huésped infectado, parcialmente conocidos o desconocidos, a los que suman su acción otros exógenos (alimentos, fármacos, etc.) incontrolables. Numerosos datos avalan que aproximadamente el $20 \%$ de la población infectada padecerá lesiones gástricas y duodenales, de las que la úlcera es la más frecuente. El desarrollo del cáncer gástrico, concretamente del tipo intestinal localizado distalmente, ha sido documentado con numerosos datos. En 1994, la Organización Mundial de la Salud declaró oficialmente a la bacteria $H p$ agente carcinógeno tipo I (26). Aunque no está consensuada la terapia erradicadora de $\mathrm{Hp}$ para prevenir el desarrollo del carcinoma gástrico, numerosos investigadores básicos y clínicos siguen esta línea de investigación: los primeros para analizar y conocer los mecanismos por los que la bacteria colabora en la oncogénesis gástrica; y los clínicos para determinar el momento de realizar el tratamiento y el beneficio de la curación de la infección para prevenir el desarrollo de las lesiones premalignas y la curación de las mismas.

Numerosos estudios han proporcionado datos discrepantes sobre la regresión de la metaplasia intestinal (MI), de la displasia y de la atrofia gástrica, lesiones angulares sobre las que crece el tumor. Señalo algunos datos prometedores. Investigadores japoneses han demostrado en cánceres gástricos inducidos experimentalmente por infección de $H p$ en gerbilos mongoles que la curación de la infección reduce la incidencia de carcinomas, tipo intestinal (27). Apoyados en estos resultados experimen- 
tales, Takenaka y cols. (28), en un ensayo sobre una muestra de 2.644 tratados con terapia erradicadora, comprobaron una reducción importante de incidencia de cáncer gástrico, particularmente del tipo intestinal, en los pacientes erradicados comparados con los que la bacteria $H p$ permanecía en la mucosa gástrica.

Un tema debatible es el momento de tratar a los pacientes infectados con $\mathrm{Hp}$ para prevenir la aparición de GA, MI y displasia, porque los resultados obtenidos de la erradicación de la bacteria son variables y contradictorios, si bien avalan la tendencia actual a tratar a los pacientes infectados para prevenir el desarrollo del cáncer gástrico en fases tempranas de la infección establecida en edades más jóvenes. En este sentido, el grupo italiano de Fuccio y cols. (29), en una revisión sistemática de estudios epidemiológicos, observacionales, de intervención y con modelos de toma de decisiones, obtienen datos a favor de una terapia erradicadora de la infección preventiva, aunque parece beneficiar solamente a un subgrupo de pacientes. El modelo de toma de decisiones apoya que el diagnóstico poblacional de $H p$ puede ser costeefectivo, aunque son necesarios ensayos con muestras de gran tamaño y un seguimiento de mayor duración para recomendar la aplicación de este programa a toda la población infectada. En otro trabajo, también de revisión, De Vries y Kuipers (30) confirman que el efecto preventivo de la erradicación de $H p$ en pacientes infectados con lesiones gástrica premalignas es dudoso, especialmente en pacientes con MI y displasia. En cambio la prevención primaria conseguida por la erradicación de $\mathrm{Hp}$ ha sido demostrada, lo que sugiere la conveniencia de terapia erradicadora preventiva en fases tempranas de carcinogénesis gástrica. Algunas guías terapéuticas, particularmente la obtenida por consenso del Grupo Europeo para estudio de Helicobacter, aconsejan considerar la erradicación del $H p$ para la prevención del cáncer gástrico (31).

¿Algunos posibles efectos colaterales pueden resultar de un programa amplio de terapia erradicadora? Bastantes voces recomiendan prudencia por el posible riesgo de aumentar la resistencia a los antibióticos, particularmente a la claritromicina y derivados nitroimidazólicos. Aunque este riesgo existe, es menor que el causado por tratamientos con estos antibióticos en otras infecciones respiratorias y urinarias de dudosa indicación. También, el predominio de pacientes con síndrome de reflujo gastroesofágico en relación con los que consultan por úlcera péptica ha sido asignado a la erradicación del $H p$. Esta relación tiene un soporte científico en un pequeño grupo de pacientes con gastritis corporal predominante.

Finalizo este editorial con mi respuesta a la pregunta del título: la bacteria $H p$ no debe convivir con la DF. Aunque no sea un factor etiológico único más que en una exigua proporción de pacientes, la infección de esta bacteria en la mucosa gástrica causa una gastritis crónica, innegable enfermedad infecciosa, con etiología, lesiones anatomopatológicas, clínica en algunos pacientes e historia natural impredecible, condicionada por otros factores, como en otras enfermedades infecciosas. Si el diagnóstico de una enfermedad infecciosa implica un tratamiento: ¿qué argumentos avalan la abstención del tratamiento erradicador de $H p$ para conseguir su desaparición en la DF? 


\section{BIBLIOGRAFÍA}

1. Sergent E, Ribadeua-Dumas L, Barbonneix L. Tratado de patología médica y de terapeútica aplicada. Traducción por F. Reig. Barcelona: Editorial Pubul; 1927.

2. Urrutia L. Enfermedades del estómago. $2^{\mathrm{a}}$ ed. Madrid: Editorial Calpe; 1925.

3. Balboa RA, Castro A, Mearin F. Concepto, definición y clasificación de las dispepsias. En: Mearin F, editor. Dispepsia tuncional. Tan desconocida como frecuente. Barcelona: Ediciones Doyma; 1997. p. 5-11.

4. Mearin F. ¿Qué se entiende por dispepsia, dispepsia orgánica y dispepsia funcional? En: Monés J, editor. Dispepsia funcional. Biblioteca básica 3. Madrid: Arán Ediciones SL; 2007. p. 15-8.

5. Talley NJ, Stanghellini V, Heading RC, Koch KL, Malagelada JR, Tytgat GNJ. Functional gastroduodenal disorders. Gut 1999; 45 (Supl.): 37-42.

6. Tack J, Talley NJ, Camilleri M, Holtman G, Hu P, Malagelada JR, et al. Functional gastroduodenal disorders. A working team report for the Rome III consensus on functionalgastrointestinal disorders. Gastroenterology 2006; 130: 1466-79.

7. Agréus L, Svârdsudo K, Nyren O, Tibblin G. Irritable bowell syndrome and dispepsia in the general population: overlap and lack of stability over time. Gastroenterology 1995; 109 (3): 671-80.

8. Lindell GH, Celebioglu F, Graffner HO. Non-ulcer dyspepsia in the long-term perspective. Eur J Gastroenterol Hepatol 1995; 7 (9): 829-33.

9. Caballero-Plasencia AM, Sofos-Kontoyannis S, Valenzuela Barranco M, et al. Irritable bowel syndrome in patients with dyspepsia: a community-based study in southern Europe. Eur J Gastroenterol Hepatol 1999; 1185: 517-22.

10. Conchillo JM, Selimah M, Bredenoord AJ, Samson M, Smouth JPM. Air swallowing, belching, acid and non-acid reflux in patients with functional dyspepsia. Alimentary Pharmacology\& Therapeutics 2007; 25: 965-71.

11. Rey Díaz-Rubio E. Aspectos clínicos de la dispepsia functional. ¿Hay alguna relación entre la dispepsia funcional y el síndrome de intestino irritable? En: Monés J, coordinador. Dispepsia Funcional. Biblioteca Básica 3. Madrid: Arán Ediciones SL; 2007. p. 15-8.

12. Collins JSA, Hamilton PW, Watt PCH, Sloan JM, Love AHG. Superficial gastritis and Campylobacter pylori in dyspeptic patients-a quatitative study using computer-linked image analysis. J Pathol 1989; 158: 303-10.

13. Loffeld RJLF, Potrees HVPJ, Arends JW, Stoberingh E, Fledrig JA. Campylobacter associated gastritis in patients with non-ulcer dyspepsia. J Clin Pathol 1988; 41: 85-8.

14. Rokkas T, Pursey C, Uzoechina E, Dorrington L, Simmons NA, Flipe MI. Non-ulcer dyspepsia and shortterm De-nol therapy: a placebo controlled trial with particular reference to the role of Campylobacter pylori. Gut 1988; 29: 1386-91.

15. Loffeld RJLF, Potters HVJP, Stobberingh E, Flendrig JA, Van Spreeeuwel JP, Arends JW. Campylobacter associated gastritis in patients with non-ulcer dyspepsia:a double blind placebo controlled trial with colloidal bismuth subcitrate. Gut 1989; 30: 1206-12.

16. Schubert TT, Schubert AB, Ma CK. Symptoms, gastritis and helicobacter pylori in patients referred for endoscopy. Gastrointest Endosc 1992; 38: 357-60.

17. Czinn SJ, Bertram TA, Murray PD, Yang P. Relationship between gastric inflammatory response and symptoms in patients infected with Helicobacter pylori. Scand J Gastroenterol 1991; 26 (Supl. 81): 33-7.

18. Pajares JM. Helicobacter pylori y dispepsia funcional: ¿huésped o patógeno? En: Mearin F, editor. Dispepsia funcional. Tan desconocida como frecuente. Barcelona: Ediciones Doyma; 1997. p. 113-6.

19. Calvet Calvo X. Fisiopatología de la dispepsia funcional. ¿Qué papel juega Helicobacter pylori? En: Monés J, coordinador. Dispepsia funcional. Biblioteca Básica 3. Madrid: Arán Ediciones SL; 2007. p. 103-15.

20. Moayyedi P, Soo S, Deeks J, Delaney B, Harris A, Innes M, et al. Eradicatuion of Helicobacter pylori for non-ulcer dyspepsia. Cochrane database Suys Rev 2006; CD 002096.

21. Helicobacter pylori in peptic ulcer disease. HIH Consensus Conference. JAMA 1994; 272 (1): 65-8.

22. Current European Concepts in the Management of Helicobacter. The Maastricht Consensus Report Gut 1997; 41: 8-13.

23. Drossman DA. The functional disorders and the Rome III process. Gastroenterology 2006; 130: 1377-90.

24. Ford AC, Forman D, Nathan J, Crocombe WD, Axon ATR, Moayyedis P. Clinical trial: knowledge of negative Helicobacter pylori status reduces subsequente dyspepsia-related resorce use. Aliment Pharmacol Ther 2007; 26: 1267-75

25. Ford AC, Forman D, Bailey AG, Axon ATR, Moayyedi P. A community screening program for Helicobacter pylori saves money: ten -year follow-up of a randomised controlled trial. Gastroenterology 2005; 129: 1910-7.

26. IARC. Monographs on the Evaluation of the Carcinogenic Risks to Human. Vol. 61. Schistosomiases, liver flukes and Helicobacter pylori. Lyon: International Agency for Research on Cancer, 1994.

27. Nozaki K, Shimizu N, Ikehara Y, et al. Effect of early eradication on Helicobacter pylori-related gastric carcinogenesis in Mongolian gerbils. Cancer Sci 2003; 94: 235-9.

28. Takenaka R, Okada H, Kato J, et al. Helicobacter pylori eradication reduced the incidence of gastric cancer, especially of the intestinal type. Aliment Pharmacol Ther 2007; 25: 805-12.

29. Fuccio L, Zagari RM, Minardi ME, Bazzoli F. Systematic review: Helicobacter pylori eradication for the prevention of gastric cancer. Aliment Pharmacol Ther 2007; 25: 133-41.

30. De Vries AC, Kuipers EJ. Review article: Helicobacter pylori eradication for the prevention og gastric cancer. Aliment Pharmacol Ther 2007; 26 (Supl. 2): 25-35.

31. Malferteiner P, Mégraud F, O'Morain C, et al. Current cepts in the management of Helicobacter pylori infection. The Maastricht -3 Consensus report. Gut 2007; 56: 772-81. 